\title{
The EFfeCts of UnCertainty on Optimal CONSUMPTiON*
}

\author{
RoBin MASON \\ Department of Economics, \\ University of Southampton \\ STEPHEN WRIGHT \\ Faculty of Economics and Politics, \\ University of Cambridge
}

30th June 1999

\begin{abstract}
When marginal utility is convex and there is pure labour income uncertainty, certain results are well-known. Asset return uncertainty is often assumed to have qualitatively similar effects; see e.g. Skinner (1988). We show that this assumption is not correct. Asset return uncertainty gives rise to an additional term in the Euler equation, which by introducing a role for current cash-in-hand, may work in the opposite direction to the precautionary motive, leading to ambiguity in the slope of the expected consumption time profile. We present a linearised version of the Euler equation, and an associated closed form solution, in order to provide intuition for these results. Numerical analysis indicates that the approximation is reasonable for empirically plausible estimates of the variances of the underlying disturbances.
\end{abstract}

AdDRESS FOR CORRESPONDENCE: Stephen Wright, Faculty of Economics and Politics, Austin Robinson Building, Sidgwick Avenue, Cambridge CB3 9DD, U.K.. Tel.: +44 1223 335234; fax.: +44 1223 335475; e-mail: stephen.wright@econ.cam.ac.uk.

Filename: PRECMOT7.tex.

*We are grateful to Donald Robertson and four anonymous referees for helpful comments. The latest draft of this paper can be found at http://www. soton.ac.uk/ ram2/papers.html. 


\section{INTRODUCTION}

Consumption models with precautionary saving (i.e. strictly convex marginal utility) are difficult to solve in general. Consequently, much of the existing literature has relied on restrictive assumptions to derive analytical results. Many papers assume that the representative asset has a return which is non-stochastic (e.g. is a 'safe' short-term government bond). See Zeldes (1989), Kimball (1990), Caballero (1991), Carroll (1991), Deaton (1991), van der Ploeg (1993), and Weil (1993), amongst many others. A certain amount has been established in this case, when the only source of uncertainty is (non-diversifiable) labour income risk. The most clear-cut result, established by Leland (1968), is that there is an upward tilt to the expected consumption time profile, so that expected future consumption is higher than it would be in the absence of the risk. Also, the form of the Euler equation implies that the expected growth in consumption is independent of current cash-in-hand.

The assumption of no asset return risk is restrictive, for two reasons. First, there is evidence (see for example Siegel (1992)) that over typical investment horizons, historic real returns on treasury bills and government bonds have been almost as risky as equities. ${ }^{1}$ Secondly, certain standard results indicate that asset return and labour income uncertainty are not equivalent in their effects on optimal consumption. For example, when the consumer is a rentier, certainty equivalence arises when utility is logarithmic (despite the presence of the precautionary motive); with only labour income risk, certainty equivalence requires quadratic utility. (See, for example, the exposition in Blanchard and Fischer (1989).) In addition, Carroll and Kimball (1996) (providing analytical proof of Zeldes' (1989) numerical results) show that labour income uncertainty alone implies that the optimal consumption function is strictly concave in wealth when the utility function exhibits hyperbolic absolute risk aversion (but is not logarithmic or quadratic).

The absence of closed form solutions means that few papers have considered both asset return and labour income uncertainty at the same time. An exception is Skinner

\footnotetext{
${ }^{1}$ Arguably the primary source of the real risk to bills, at least historically, has arisen from inflation uncertainty. Understanding the role of real asset return uncertainty is therefore a prerequisite for e.g. any attempt to analyse the implications of inflation uncertainty.
} 
(1988), who develops an approximate solution for the optimal consumption function in this case. His analysis implies that asset return uncertainty is qualitatively equivalent to pure labour income uncertainty. We show below that this implication is incorrect. Campbell (1993) and Campbell and Viceira (1998) also develop an approximate solution, this time by using a log-linear expansion of the budget constraint, assuming that the variation in the consumption-wealth ratio is small. This approach does not allow an explicit comparison of the different effects of the two types of uncertainty. Talmain (1998) and Binder et al. (1998) develop approximations for general income and asset processes in order to facilitate numerical analysis.

In contrast, this paper provides both general and approximate analytical results and direct intuition for the fact that asset return and labour income uncertainty have different qualitative and quantitative effects on optimal consumption.

The analysis identifies three different ways in which asset return risk affects optimal consumption. First, it increases the tendency for prudence in a way which is qualitatively similar to the case of pure labour income uncertainty. In the latter case, the precautionary motive is proportional to the variance of the labour income process; with asset return uncertainty as well, the precautionary motive is proportional to the sum of labour income variance and (a term in) asset return variance. Secondly, it introduces a qualitatively different 'hazard effect', which implies that a consumer with net savings, faced with an intertemporal budget constraint which is made risky by asset return uncertainty, engages in less intertemporal consumption smoothing. Thirdly, it causes a 'precautionary covariance' effect. The hazard effect arises because marginal utility is downward sloping (i.e. the consumer is risk averse); the precautionary covariance effect offsets or adds to this effect, according to whether consumption is expected to rise or fall in the future, because the slope of marginal utility becomes less negative as its argument increases (i.e. the consumer is prudent).

In addition to these three effects, asset return risk introduces a role for cash-inhand in determining the expected growth in consumption (absent when there is only labour income risk). Consequently, since the level of wealth is serially correlated, consumption no longer follows a random walk (contrary to Hall's (1978) hypothesis).

Skinner (1988) uses a second order Taylor approximation to the Euler equation to 
derive optimal consumption when there is both labour income and asset return risk. Skinner's solution has been used widely as the basis for empirical studies; see, for example, Miles (1997). We use a similar approach to Skinner's, but come to rather different conclusions. The reason for this difference is that (as Skinner acknowledges) his method does not genuinely solve even the approximate problem, since in order to make the problem soluble, he exogenises a potentially important linkage between asset return uncertainty and the relationship between consumption in adjacent periods, via the intertemporal budget constraint. It is this restriction which ensures that asset return and labour income uncertainty are qualitatively equivalent in Skinner's analysis. Our approximation, in contrast to Skinner's, endogenises the impact of asset return uncertainty on the optimal consumption path.

The advantage of our approach is that it captures the qualitative difference between labour income and asset return risk for optimal consumption. The price paid for this is the strength of the assumptions required: that the optimal deviations of consumption and assets from the risk-free paths are sufficiently small in percentage terms. This will not always be the case (for example, if the labour income process has high variance). Comparison with exact numerical simulations suggests, however, that the assumptions are reasonable for empirically plausible values of the variances of the underlying disturbances.

The structure of the paper is as follows: the next section discusses the general Euler equation. Section 3 approximates this equation with a second order Taylor expansion. Section 4 approximates further to a linear Euler equation and develops a closed form solution for optimal consumption. Section 5 considers some of the assumptions used in the approximation. Section 6 performs numerical analysis of the approximation and an exact solution. Section 7 concludes. The appendix contains technical details of some of the arguments in the main text. 


\section{The General Euler Equation}

\subsection{The Model}

The problem is set up in standard form. A consumer chooses consumption in each period of life, $t=0,1, \ldots, T$, to maximise the present discounted value of an expected flow of instantaneous utilities:

$$
V_{t}=\max _{\left\{c_{t+i}\right\}} \sum_{i=0}^{T-t} \delta^{-i} \mathbb{E}_{t}\left[U\left(c_{t+i}\right)\right]
$$

where $\delta>1$ is the consumer's discount factor; $c_{t}$ is consumption at time $t$; and the instantaneous per period utility (or felicity) function $U$ enters in additive separable form, and is assumed to be continuously differentiable. $\mathbb{E}_{t}$ represents the expectations operator, evaluated given information at time $t$. (The time subscript will be dropped subsequently where its omission does not cause confusion.) The consumer has two sources of income, labour income and returns on net assets, which are assumed to be perfectly fungible. $y_{t}$ is labour income; $a_{t}$ is net assets; $x_{t}=a_{t}+y_{t}$ is called 'cashin-hand'. Borrowing is not ruled out: $a_{t}$ may be positive or negative. We follow past literature in treating labour income as, effectively, a stochastic endowment; hence the labour supply decision is ignored.

Consumption in any period is chosen after asset returns and labour income are realised. There is only one asset, which yields a return in period $t+1$ of $r_{t+1}$ for an investment of one unit in period $t$. The consumer's assets therefore evolve according to the equation

$$
\begin{aligned}
a_{t+1} & =\left(a_{t}+y_{t}-c_{t}\right) r_{t+1} \\
\text { or } \quad x_{t+1} & =\left(x_{t}-c_{t}\right) r_{t+1}+y_{t+1}
\end{aligned}
$$

The expected asset return $\mathbb{E}_{t}\left[r_{t+1}\right]=\rho$ for all $t$. It is assumed that $\rho=\delta$ i.e. there is no relative impatience; this assumption is reviewed in section 5. It is also assumed that $r_{t}$ and $y_{t}$ are serially uncorrelated and independent stochastic processes. (Further assumptions about these processes are made in the next section, and the assumptions 
reviewed in section 5.)

The lifetime budget constraint is

$$
a_{T}+y_{T}-c_{T} \geq 0
$$

i.e. terminal net assets cannot be negative. Since no bequest motive is included in the model, this means that $c_{T}=x_{T}$ when the constraint binds. ${ }^{2}$

The maximisation in equation (1), subject to the asset evolution equation (2) and the lifetime budget constraint (3), yields the usual Euler equation at time t:

$$
\begin{aligned}
U^{\prime}\left(c_{t}\right) & =\mathbb{E}\left[\frac{r_{t+1}}{\delta} U^{\prime}\left(c_{t+1}\right)\right] \\
& =\mathbb{E}\left[U^{\prime}\left(c_{t+1}\right)\right]+\frac{1}{\delta} \operatorname{Cov}\left[U^{\prime}\left(c_{t+1}\right), r_{t+1}\right]
\end{aligned}
$$

\subsection{Pure Labour Income Uncertainty and the Euler Equation}

When there is no asset return uncertainty, the covariance term in equation (4) will be zero. In this case, with strictly convex marginal utility, the familiar result that the expected consumption time profile is tilted upwards emerges (by Jensen's inequality, $\mathbb{E}\left[U^{\prime}\left(c_{t+1}\right)\right]>U^{\prime}\left(\mathbb{E}\left[c_{t+1}\right]\right)$, and hence $\left.c_{t}<\mathbb{E}\left[c_{t+1}\right]\right)$.

\subsection{Asset Return Risk and the Euler Equation}

When asset return risk enters the picture, the outcome is more complicated. Equation (4) can be written as

$$
U^{\prime}\left(c_{t}\right)=U^{\prime}\left(\mathbb{E}\left[c_{t+1}\right]\right)+J+\frac{1}{\delta} \operatorname{Cov}\left[U^{\prime}\left(c_{t+1}\right), r_{t+1}\right]
$$

where $J \equiv \mathbb{E}\left[U^{\prime}\left(c_{t+1}\right)\right]-U^{\prime}\left(\mathbb{E}\left[c_{t+1}\right]\right) \geq 0$, a Jensen's inequality term. The sign and magnitude of the covariance term, relative to $J$, therefore determine the slope of the

\footnotetext{
${ }^{2}$ We do not attempt to take account of two important issues: the effect of a stochastic date of death (see e.g. Blanchard (1985)); and the existence of liquidity constraints which impede or prevent borrowing (see e.g. Deaton (1991)).
} 
expected optimal consumption profile. With minor assumptions, certain aspects of both can be determined; and it can be shown that asset return risk implies a time profile of expected consumption which, in contrast to the case of pure labour income risk, may be either positively or negatively sloped. In the appendix, it is shown formally that the covariance term has the opposite sign to $\left(x_{t}-c_{t}\right)$ (see lemma A.1). The intuition is straightforward. A high level of realised asset return in period $t+1$ leads (other things being equal) ${ }^{3}$ to a high level of cash-in-hand in period $t+1$ if the consumer is a net saver. Consumption in period $t+1$ rises as a result; marginal utility falls. Hence marginal utility and asset returns co-vary negatively when $\left(x_{t}-c_{t}\right)>0$.

Therefore the covariance term and $J$ are of opposite sign when period $t$ saving is positive. This sign difference is interesting, in the sense that the sum of the two terms may be positive or negative. If utility is quadratic, and hence marginal utility is linear, $J=0$ and the covariance term is unambiguously negative (for positive saving). $J>0$ for convex marginal utility, and increases with the degree of convexity. Conversely, the covariance term is always negative, but becomes less negative as marginal utility becomes more convex. This indicates that there will be (non-quadratic) cases in which the covariance term is dominated by the Jensen's inequality term, as well as a borderline case where the two terms balance exactly. A trivial example of the former is when there is no net saving in period $t$; an example of the latter is a rentier with logarithmic utility. If net saving is negative, then the covariance and Jensen's inequality term are both positive. (These results are shown formally in the lemmas in the appendix.)

It is also worth noting another important feature once asset return risk is present. The impact of the covariance term is to introduce a role for current cash-in-hand into the Euler equation, which is absent both in the risk-free case and when there is only labour income risk. Thus, while the riskiness of the income profile affects the slope of the optimal consumption profile with pure labour income risk, the income profile itself does not matter, either in expectation or realisation. With asset return risk it does. Since cash-in-hand is serially correlated, this means that consumption will no longer follow a random walk, in contrast to Hall's (1978) hypothesis.

\footnotetext{
${ }^{3}$ The 'other things being equal' statement is valid, since it is assumed that $r_{t+1}$ and $y_{t+1}$ are independent random variables.
} 
In order to go further with the analysis, the general Euler equation (4) must be approximated. This is done in the next two sections, in which only second order (section 3) and first order (section 4) terms are considered.

\section{A Quadratic Approximation of the Euler Equation}

In this section, a second order Taylor series approximation of the Euler equation (4) is derived. The analytical approach adopted is to approximate the equation around the riskless solution with no asset return or labour income risk. Let $c_{t}^{*}$ and $x_{t}^{*}$ denote consumption and cash-in-hand when the agent faces no risk. These can be determined quite easily; in particular, it is straightforward to show that $c_{t}^{*}$ is constant over time, and given by

$$
c^{*}=\left(\frac{\theta}{1+\theta}\right)\left(\frac{1}{1-(1+\theta)^{-(T-t+1)}}\right)\left(x_{t}^{*}+\sum_{i=0}^{T-t} \frac{1}{(1+\theta)^{i}} \hat{y}_{t+i}\right)
$$

where $1+\theta=\delta$ and $\hat{y}_{t+i}$ is the time $t$ expectation of labour income at time $t+i$.

The left hand side and the first term on the right hand side of equation (4) can be approximated using a second order Taylor series expansion around $c^{*}$. The terms in equation (4) become

$$
\begin{aligned}
U^{\prime}\left(c_{t}\right) & \approx U^{\prime}+U^{\prime \prime}\left(c_{t}-c^{*}\right)+\frac{U^{\prime \prime \prime}}{2}\left(c_{t}-c^{*}\right)^{2} \\
\mathbb{E}\left[U^{\prime}\left(c_{t+1}\right)\right] & \approx U^{\prime}+U^{\prime \prime} \mathbb{E}\left[c_{t+1}-c^{*}\right]+\frac{U^{\prime \prime \prime}}{2} \mathbb{E}\left[\left(c_{t+1}-c^{*}\right)^{2}\right]
\end{aligned}
$$

where all derivatives are evaluated at $c_{t}=c^{*}$. Then

$$
\begin{aligned}
U^{\prime}+U^{\prime \prime}\left(c_{t}-c^{*}\right)+\frac{U^{\prime \prime \prime}}{2}\left(c_{t}-c^{*}\right)^{2} \approx & U^{\prime}+U^{\prime \prime} \mathbb{E}\left[c_{t+1}-c^{*}\right]+\frac{U^{\prime \prime \prime}}{2} \mathbb{E}\left[\left(c_{t+1}-c^{*}\right)^{2}\right] \\
& +\frac{1}{\delta} \operatorname{Cov}[., .] .
\end{aligned}
$$

Dividing all terms in equation (6) through by $U^{\prime \prime}$ and $c^{*}$ gives an expression in terms 
of percentage deviations from $c^{*}$ :

$$
\begin{aligned}
\frac{c_{t}-c^{*}}{c^{*}}-\frac{(1+\gamma)}{2}\left(\frac{c_{t}-c^{*}}{c^{*}}\right)^{2} \approx & \mathbb{E}\left[\frac{c_{t+1}-c^{*}}{c^{*}}\right]-\frac{(1+\gamma)}{2} \mathbb{E}\left[\left(\frac{c_{t+1}-c^{*}}{c^{*}}\right)^{2}\right] \\
& +\frac{1}{\delta U^{\prime \prime}} \frac{\operatorname{Cov}[., .]}{c^{*}}
\end{aligned}
$$

where $\frac{U^{\prime \prime \prime} c^{*}}{U^{\prime \prime}}$ is set equal to $-(1+\gamma)$. In the special case that utility is CRRA, $\gamma$ is the constant coefficient of relative risk aversion. The case of risk neutrality $(\gamma=0)$ is ruled out.

Equation (7) can be simplified further. First, let upper case letters denote percentage differences from the riskless consumption level:

$$
C_{t}=\frac{c_{t}-c^{*}}{c^{*}}, \quad X_{t}=\frac{x_{t}-c^{*}}{c^{*}}, \quad X_{t}^{*}=\frac{x_{t}^{*}-c^{*}}{c^{*}}, \quad Y_{t}=\frac{y_{t}-c^{*}}{c^{*}} .
$$

The following assumptions are made about the stochastic processes in the model. $r_{t}$ is assumed to be conditionally normally distributed. Its mean, already specified, is $\rho$, and its variance is $\sigma_{r}^{2}$ :

$$
r_{t} \sim N\left(\rho, \sigma_{r}^{2}\right)
$$

Labour income $y_{t}$ is also assumed conditionally normally distributed, with mean $\hat{y}_{t}$ and variance $\sigma_{y}^{2}$ :

$$
y_{t} \sim N\left(\hat{y}_{t}, \sigma_{y}^{2}\right)
$$

(This representation is equivalent to assuming inelastic labour supply, with a stochastic real wage.) Hence $Y_{t} \sim N\left(\hat{Y}_{t}, \sigma_{Y}^{2}\right)$ where $\hat{Y}_{t}=\frac{\hat{y}_{t}-c^{*}}{c^{*}}$ and $\sigma_{Y}^{2}=\frac{\sigma_{y}^{2}}{c^{* 2}}$. Recall that asset returns and labour income are assumed also to be serially uncorrelated and independent. These assumptions are discussed in section 5 .

Next, write $\mathbb{E}\left[C_{t+1}^{2}\right]=\operatorname{Var}\left[C_{t+1}\right]+\left(\mathbb{E}\left[C_{t+1}\right]\right)^{2}$. The variance of $C_{t+1}$ can be determined using the Delta method (see e.g. Greene (1993), p. 297). $C_{t+1}$ is some (as yet undetermined) function of cash-in-hand $X_{t+1}$; call this function $g\left(X_{t+1}\right)=$ 
$g\left(\left(X_{t}-C_{t}\right) r_{t+1}+Y_{t+1}\right)$. The Delta method shows that the function $g($.$) is asymptot-$ ically normally distributed with variance

$$
\left(\frac{d g}{d X_{t+1}}\right)^{2}\left(\left(X_{t}-C_{t}\right)^{2} \sigma_{r}^{2}+\sigma_{Y}^{2}\right)
$$

Using notation that will become apparent in section 4 , let $\frac{d g}{d X_{t+1}} \equiv \zeta_{0, t+1}$. Note that (i) $\zeta_{0, t+1}$ is in general a function of $X_{t+1}$; (ii) it is positive (this is shown formally in lemma A.1 in the appendix).

The covariance term is derived as a further Taylor series approximation, which can be shown (again using the Delta method, and the assumption of independent, normally distributed disturbances) to be:

$$
\operatorname{Cov}\left[U^{\prime}\left(c_{t+1}\right), r_{t+1}\right] \approx c_{t}^{*} \zeta_{0, t+1}\left(X_{t}-C_{t}\right) \sigma_{r}^{2}\left(U^{\prime \prime}+U^{\prime \prime \prime} c^{*} \mathbb{E}\left[C_{t+1}\right]\right)
$$

Equation (7) can then be written in terms of deviations from the riskless solution as

$$
\begin{aligned}
C_{t}-\frac{(1+\gamma)}{2} C_{t}^{2} \approx & \mathbb{E}\left[C_{t+1}\right]-\frac{(1+\gamma)}{2}\left(\mathbb{E}\left[C_{t+1}\right]\right)^{2} \\
& -\frac{(1+\gamma)}{2} \zeta_{0, t+1}^{2}\left(\sigma_{Y}^{2}+\left(X_{t}-C_{t}\right)^{2} \sigma_{r}^{2}\right) \\
& +\zeta_{0, t+1}\left(X_{t}-C_{t}\right) \frac{\sigma_{r}^{2}}{\delta}\left(1-(1+\gamma) \mathbb{E}\left[C_{t+1}\right]\right)
\end{aligned}
$$

Equation (11) is a quadratic, and so detailed analysis (such as comparative statics) is difficult. The equation does show, however, the qualitative effect of asset return and labour income risk. The equation can be rewritten as

$$
\begin{aligned}
f\left(C_{t}\right) & =f\left(\mathbb{E}\left[C_{t+1}\right]\right)+\text { Precautionary }+ \text { Covariance, } \\
\text { where } f(C) & \equiv C-\frac{(1+\gamma)}{2} C^{2}, \\
\text { Precautionary } & =-\frac{(1+\gamma)}{2} \zeta_{0, t+1}^{2}\left(\sigma_{Y}^{2}+\left(X_{t}-C_{t}\right)^{2} \sigma_{r}^{2}\right) \leq 0, \\
\text { Covariance } & =\zeta_{0, t+1}\left(X_{t}-C_{t}\right) \frac{\sigma_{r}^{2}}{\delta}\left(1-(1+\gamma) \mathbb{E}\left[C_{t+1}\right]\right) .
\end{aligned}
$$


The precautionary term is familiar from standard analysis of the consumption problem (see e.g. Deaton (1992)), and is consistent with our more general analysis above. When marginal utility is convex $(\gamma>-1)$, this term is unambiguously negative for non-zero risk (either $\sigma_{Y}$ or $\sigma_{r}$ greater than zero). Consequently, the precautionary term on its own would mean that $C_{t}<\mathbb{E}\left[C_{t+1}\right]$ i.e. the consumption profile is upward sloping. When $\sigma_{Y}>0$ and $\sigma_{r}=0$, the term depends only on $\gamma, \sigma_{Y}$ and $\zeta_{0, t+1}$. Asset return risk $\left(\sigma_{r}>0\right)$ increases the precautionary term in absolute size, but also makes it dependent on the (endogenous) variables $X_{t}$ and $C_{t}$.

Since the terms in labour income and asset return risk in the total expression for the precautionary motive are additive, some previous research has assumed that the two types of risk are qualitatively equivalent; see, in particular, Skinner (1988). This conclusion ignores the covariance term, which depends only on asset return risk. This term is positive when saving is positive i.e. $\left(X_{t}-C_{t}\right)>0$; where the precautionary term is zero, therefore, covariance would lead to a downward sloping consumption profile $\left(C_{t}>\mathbb{E}\left[C_{t+1}\right]\right)$ in this case. The covariance term has two components. The first, equal to $\zeta_{0, t+1}\left(X_{t}-C_{t}\right) \frac{\sigma_{r}^{2}}{\delta}$, is positive when saving is positive, $\left(X_{t}-C_{t}\right)>0$. This is a 'hazard effect': it reflects the riskiness of smoothing consumption across the life cycle when the rate of return of the asset is uncertain. The possibility that returns may be zero in the next period effectively increases the consumer's discount rate, leading to a shift in consumption from the future to the present - or, equivalently, a reduction in consumption smoothing. The hazard effect works in the opposite direction when the consumer is a borrower i.e. $\left(X_{t}-C_{t}\right)<0$; but the outcome is again that of a reduction in consumption smoothing.

The second component, a 'precautionary covariance effect', is equal to $-(1+$ $\gamma) \zeta_{0, t+1}\left(X_{t}-C_{t}\right) \frac{\sigma_{r}^{2}}{\delta} \mathbb{E}\left[C_{t+1}\right]$. This effect is zero when marginal utility is linear. When $\gamma>-1$, the sign of the effect is determined by the sign of $\left(X_{t}-C_{t}\right) \mathbb{E}\left[C_{t+1}\right]$. When saving is positive and the next period's consumption is expected to be above the riskless level, the precautionary covariance effect offsets the hazard effect (although lemma A.1 in the appendix shows that this total covariance term is always positive). When saving is positive, but next period's consumption is expected to be below the riskless level, the precautionary covariance effect reinforces the hazard effect. 


\section{A Linear Solution for Optimal Consumption}

In this section, the quadratic Euler equation (11) is linearised, and a closed form solution for optimal consumption is developed.

\subsection{A Linear Approximation of the Euler Equation}

The linearisation relies on the following assumption:

Assumption: Percentage deviations from the riskless solution are small:

$$
C_{t}^{2},\left(\mathbb{E}\left[C_{t+1}\right]\right)^{2},\left(X_{t}-X_{t}^{*}\right)^{2}, C_{t}\left(X_{t}-X_{t}^{*}\right),\left(X_{t}-X_{t}^{*}\right) \mathbb{E}\left[C_{t+1}\right], C_{t} \mathbb{E}\left[C_{t+1}\right] \approx 0
$$

This assumption essentially requires that the standard deviations of the asset return and labour income distributions are small (i.e. 'risk' is sufficiently 'low'); and that marginal utility is not too convex. Notice that the assumption does not require that $X_{t}^{2} \approx 0$ : cash-in-hand may deviate significantly from zero over the life cycle. It does require that the difference in cash-in-hand between the risky and riskless case be sufficiently small. Even this may be strong; see e.g. Skinner (1988) for estimates of the size of precautionary savings. We maintain this assumption in order to derive a linear solution for optimal consumption which captures all relevant effects (see below).

The assumption can be used to eliminate the terms in $C_{t}^{2}$ and $\left(\mathbb{E}\left[C_{t+1}\right]\right)^{2}$ in equation (11), since both will be approximately zero. The term involving $\left(X_{t}-C_{t}\right)^{2}$ must be expanded further (Skinner's (1988) approach is to treat the term simply as a constant):

$$
\begin{aligned}
\left(X_{t}-C_{t}\right)^{2} & =X_{t}^{2}-2 X_{t} C_{t}+C_{t}^{2} \\
& =\left(X_{t}-X_{t}^{*}\right)^{2}+C_{t}^{2}-X_{t}^{* 2}+2 X_{t}\left(X_{t}^{*}-C_{t}\right) \\
& =\left(X_{t}-X_{t}^{*}\right)^{2}+C_{t}^{2}-X_{t}^{* 2}+2 X_{t}^{*}\left(X_{t}-C_{t}\right)-2 C_{t}\left(X_{t}-X_{t}^{*}\right) .
\end{aligned}
$$

The 'small percentage deviations' assumption in equation (12) implies that

$$
\left(X_{t}-C_{t}\right)^{2} \approx 2 X_{t}^{*}\left(X_{t}-C_{t}\right)-X_{t}^{* 2}
$$


Finally, note that $\left(X_{t}-C_{t}\right) \mathbb{E}\left[C_{t+1}\right]=\left(\left(X_{t}-X_{t}^{*}\right)-\left(C_{t}-X_{t}^{*}\right)\right) \mathbb{E}\left[C_{t+1}\right]$. One further application of equation (12) allows this term to be simplified, so that equation (11) becomes

$$
\begin{aligned}
C_{t} & =\beta_{1 t} \mathbb{E}\left[C_{t+1}\right]+\beta_{2 t} X_{t}+\beta_{3 t}, \\
\beta_{1 t} & =\left(\frac{1-\sigma_{r}^{2} \Lambda_{t}}{1+\sigma_{r}^{2} \Gamma_{t}}\right), \\
\beta_{2 t} & =\left(\frac{\sigma_{r}^{2} \Gamma_{t}}{1+\sigma_{r}^{2} \Gamma_{t}}\right), \\
\beta_{3 t} & =-\frac{(1+\gamma) \zeta_{0, t+1}^{2}}{2\left(1+\sigma_{r}^{2} \Gamma_{t}\right)}\left(\sigma_{Y}^{2}-\sigma_{r}^{2} X_{t}^{* 2}\right), \\
\Lambda_{t} & =\frac{(1+\gamma) \zeta_{0, t+1} X_{t}^{*}}{\delta}, \\
\Gamma_{t} & =\zeta_{0, t+1}\left(\frac{1}{\delta}-(1+\gamma) \zeta_{0, t+1} X_{t}^{*}\right) .
\end{aligned}
$$

The linear equation (13) makes explicit the functional dependence of optimal current consumption on (i) next period's expected consumption, (ii) current cash-inhand, and (iii) cash-in-hand $X_{t}^{*}$ in the riskless case and the derivative of consumption $\zeta_{0, t+1} \equiv \frac{\partial C_{t+1}}{\partial X_{t+1}}$.

Equation (13) effectively linearises marginal utility; but it does so in a way that retains at least some of the dependence of optimal consumption on uncertainty. The coefficients in the expression depend explicitly on $\sigma_{r}$ and $\sigma_{Y}$. When there is no asset return risk $\left(\sigma_{r}=0\right), \beta_{1 t}=1, \beta_{2 t}=0$, and $\beta_{3 t}=-\frac{(1+\gamma) \sigma_{Y}^{2} \zeta_{0, t+1}^{2}}{2}<0$. This yields the familiar result with convex marginal utility: labour income uncertainty alone induces an upward tilt to the expected consumption profile. There is a bias in the linear approximation, however, due to the omission of squared deviation terms. In some restricted cases it may be possible to identify both the sign and order of magnitude of this bias.

The dependence of the coefficients on riskless cash-in-hand $X_{t}^{*}$ means that the linear equation implies that there are life cycle effects on optimal consumption under uncertainty. When $X_{t}^{*}=0$ and both asset return and labour income uncertainty are present, $0<\beta_{1 t}<1,0<\beta_{2 t}<1$, and $\beta_{3 t}<0$. The general dependence of the 
coefficients on $X_{t}^{*}=0$ is complicated by the fact that the derivative $\zeta_{0, t+1}$ is yet to be determined (this is done in the next section). The expression there (equation (15) shows that $\frac{\partial \zeta_{0, t+1}}{\partial X_{t}^{*}}=0 .{ }^{4}$ This means that

$$
\frac{\partial \beta_{1 t}}{\partial X_{t}^{*}}<0, \quad \frac{\partial \beta_{2 t}}{\partial X_{t}^{*}}>0, \quad \frac{\partial \beta_{3 t}}{\partial X_{t}^{*}}>0
$$

when $\sigma_{r}$ and $\sigma_{Y}$ are greater than zero. These derivatives mean that, as riskless cash-inhand increases, the dependence of current consumption on (i) expected consumption in the next period decreases, and (ii) current cash-in-hand increases; and that the general level of current consumption increases.

\subsection{The Closed Form Solution}

Consider the following trial solution for the linearised Euler equation (13): ${ }^{5}$

$$
C_{t}=\zeta_{0, t} X_{t}+\sum_{i=1}^{T-t} \zeta_{i, t} \hat{Y}_{t+i}+\kappa_{t}
$$

for $t \leq T-1$. The $\left\{\zeta_{i, t}\right\}_{t=1, \ldots, T-1 ; i=1, \ldots, T-t}$ are constants, not functions of endogenous variables. Notice that the trial solution assumes that $A_{t}$ and $Y_{t}$ have the same impact on current consumption i.e. that money is fungible between assets and income.

The budget constraint and lack of bequests imply that $C_{T}=X_{T}$, and hence that $\zeta_{0, T}=1$ and $\kappa_{T}=0$. Substitution of the trial solution into equation (13) and backward recursion from the terminal period yields a solution of the same form as

\footnotetext{
${ }^{4}$ The partial derivative here should be interpreted as the change in $\zeta_{0, t+1}$ with a change in $X_{t}^{*}$ holding all $X_{t+i}^{*}, i \geq 1$ constant.

${ }^{5}$ Although the trial solution is expressed in terms of percentage differences from $c^{*}$, an equivalent expression in levels could be written. The form chosen is more appropriate, given our approach of approximating around the riskless solution.
} 
the trial solution, with recursive definitions of the coefficients:

$$
\begin{aligned}
\zeta_{0, t}= & \frac{\mu \zeta_{0, t+1}-\nu_{t} \zeta_{0, t+1}^{2}}{1+\mu \zeta_{0, t+1}-\nu_{t} \zeta_{0, t+1}^{2}}, \\
\zeta_{i+1, t}= & \left(1-\zeta_{0, t}\right)\left(1-\Phi_{t} \zeta_{0, t+1}\right) \zeta_{i, t+1}, \quad i=0, \ldots, T-t-1 \\
\kappa_{t}= & \left(1-\zeta_{0, t}\right)\left(1-\Phi_{t} \zeta_{0, t+1}\right) \kappa_{t+1} \\
& -\left(1-\zeta_{0, t}\right)\left(\frac{(1+\gamma)}{2} \zeta_{0, t+1}^{2}\left(\sigma_{Y}^{2}-X_{t}^{* 2} \sigma_{r}^{2}\right)\right)
\end{aligned}
$$

where $\mu=\frac{\rho^{2}}{\delta}+\frac{\sigma_{r}^{2}}{\delta}, \nu_{t}=\left(1+\frac{\rho}{\delta}\right)\left(1+\gamma_{t}\right) \sigma_{r}^{2} X_{t}^{*}$, and $\Phi_{t}=\frac{\sigma_{r}^{2}}{\delta}\left(1+\gamma_{t}\right) X_{t}^{*}$.

The recursive solution, although rather unwieldy, can nonetheless be used to derive some general features of the closed form. Each of equations (15)-(17) will be examined in turn, before moving on to special cases.

Equation (15) describes how the coefficient on current cash-in-hand in the linear consumption function (14) evolves over time. Notice that the equation does not involve $\sigma_{Y}$ : labour income uncertainty does not affect $\zeta_{0, t}$. This property can be used to simplify the closed form considerably when $\sigma_{r}$ is zero. Let $\zeta_{0, t}^{*}$ denote the coefficient in this case. Then, since

$$
\zeta_{0, t}^{*}=\frac{\delta \zeta_{0, t+1}^{*}}{1+\delta \zeta_{0, t+1}^{*}} \Rightarrow \zeta_{0, t}^{*}=\frac{\delta^{T-t-1}}{\sum_{j=0}^{T-t-1} \delta^{j}} .
$$

This equation can be manipulated to produce the expression for riskless consumption $c^{*}$ given in equation (5). Since $\zeta_{0, T}=1, \zeta_{0, T-1}=\frac{\mu-\nu_{T-1}}{1+\mu-\nu_{T-1}}<1$. Consequently, $\zeta_{0, t}<\zeta_{0, t+1} \forall t$ : the coefficients are increasing over time. When $\gamma=-1$ (quadratic utility),

$$
\zeta_{0, t}=\frac{\mu \zeta_{0, t+1}}{1+\mu \zeta_{0, t+1}}
$$

In this case, $\frac{\partial \zeta_{0, t}}{\partial \sigma_{r}^{2}}>0$, so that $\zeta_{0, t} \geq \zeta_{0, t}^{*} \forall t$. For higher values of $\gamma$, the situation is more complex.

Equation (16) defines the coefficients on future expected labour income at any point in time. It is evident from the form of the equation that the same factors that 
determine the $\left\{\zeta_{0, t}\right\}_{t=1}^{T}$ determine all of the coefficients. Hence, again, labour income uncertainty does not affect the value of any of the coefficients. When $\sigma_{r}=0$ or $\gamma=-1, \Phi_{t}=0$, so that

$$
\zeta_{i+1, t}=\left(1-\zeta_{0, t}\right) \zeta_{i, t+1}, \quad i=0, \ldots, T-t-1
$$

As $\zeta_{0, t} \leq 1 \forall t, \zeta_{i+1, t} \leq \zeta_{i, t+1} \forall i, t$

The intuition for equation (17), which defines $\kappa_{t}$, can be seen most easily when interest rates are non-stochastic. In this case, the equation simply cumulates the period-specific constants in (13) backwards from the terminal period.

In order to gain further insight into the impact of labour income and asset return uncertainty, it is helpful to examine some special cases.

\subsection{No Asset Return Risk}

When $\sigma_{r}=0$, the relative weighting on current cash-in-hand and future labour income is the same as in the risk-free case. This property is also evident from the linearised Euler equation (13), in which the time profile of cash-in-hand has no impact on the solution when there is no asset return risk. Hence, given the definitions of $c^{*}$ (see equation (5)) and of the percentage deviations, it is straightforward to show in this case that the closed form expression for the optimal deviation of consumption from the risk-free case is

$$
C_{t}=\kappa_{t} \equiv-k_{t} \sigma_{Y}^{2}
$$

Equation (18) confirms that with only labour income risk, there is an upward tilt in the consumption profile when utility is non-quadratic: when $\gamma>-1, k_{t}>0 ; \sigma_{Y}^{2}>0$ means that optimal current consumption is less than the riskless level, for all $t$.

\subsection{Quadratic Utility}

The coefficients in the consumption function in equation (14) are particularly 
simple in the case of quadratic utility. When $\gamma=-1$, the precautionary motive disappears. ${ }^{6}$ Equation (14) is exact in this case, since the Euler equation is linear. Simplification yields

$$
\begin{gathered}
C_{t}=\zeta_{q} \omega_{t}\left(X_{t}+\sum_{i=1}^{T-t}\left(1-\zeta_{q}\right)^{i} \hat{Y}_{t+i}\right), \\
\zeta_{q}=\frac{\mu-1}{\mu}, \quad \omega_{t}=\frac{1}{\left(1-\mu^{-(T-t+1)}\right)},
\end{gathered}
$$

which can also be written in absolute levels (rather than percentage deviations from $\left.c^{*}\right)$ as

$$
c_{t}=\left(\frac{\theta+s}{1+\theta+s}\right) \omega_{t}\left(x_{t}+\sum_{i=1}^{T-t} \frac{1}{(1+\theta+s)^{i}} \hat{y}_{t+i}\right)
$$

where $1+\theta=\delta$, and $s=\frac{\sigma_{r}^{2}}{\delta}$. Written in this form, it is evident that when $\sigma_{r}=$ 0 , the familiar certainty-equivalent solution to the permanent income problem with quadratic utility emerges. For $\sigma_{r}>0$, the form of the solution is identical to that determining $c^{*}$ in equation (5), but with a constant term added to the discount rate. This is the hazard effect, identified in section 3, in its clearest form. In the limit as $\sigma_{r}^{2}$ goes to infinity, ${ }^{7}$ this effect becomes so large that the 'pure Keynesian' case of $c_{t}=y_{t}$ emerges. Thus asset return uncertainty here parallels the well-known effect of imperfect capital markets, but in a way which is symmetric between borrowers and lenders.

\subsection{A Rentier with Small Risk}

Consider the optimal consumption problem of an agent who receives no labour income, but earns returns from a risky asset. To simplify the case further, assume that the standard deviation of asset returns, $\sigma_{r}$, is sufficiently small that $\zeta_{0, t+1} \approx \zeta_{0, t+1}^{*}$;

\footnotetext{
${ }^{6}$ An alternative rationale, which would not depend on the restrictive quadratic assumption, would be to assume that $X_{t}^{*}$ is sufficiently small for all such terms to be neglected. An example of this would be the case of someone with labour income which fluctuated around a stable average, and who therefore has relatively low net assets.

${ }^{7}$ Since the solution is exact in the quadratic case, it is valid to take the limit.
} 
and that $X_{t} \approx X_{t}^{*}$. (These assumptions are used only in this subsection in order to provide intuition.) Write equation (13) as

$$
C_{t}=v_{1 t} \mathbb{E}\left[C_{t+1}\right]+v_{2 t}
$$

Then the definitions of the closed form coefficients in equations (15)-(17) mean that

$$
v_{1 t}=\frac{\delta^{2}-(1+\gamma) \sigma_{r}^{2}}{\delta^{2}-\gamma \delta \zeta_{0, t+1}^{*} \sigma_{r}^{2}}, \quad v_{2 t}=\frac{(1-\gamma) \sigma_{r}^{2}}{2 \delta\left(\delta-\gamma \zeta_{0, t+1}^{*} \sigma_{r}^{2}\right)}
$$

When $\sigma_{r}$ is sufficiently small, $v_{1 t} \in(0,1)$. By construction, $\delta-\gamma \zeta_{0, t+1}^{*} \sigma_{r}^{2}>0$. Therefore when $\gamma=-1, v_{2 t}>0$. It is straightforward to show that $\frac{\partial v_{2 t}}{\partial \gamma}<0$. Consequently, the intercept term is positive for low levels of prudence, but eventually becomes negative as $\gamma$ increases.

The less prudent rentier (with a low $\gamma$ ) therefore has a downward sloping consumption profile. In the terminology of the section 3, the 'hazard effect' dominates when the rentier has a low $\gamma$. As the rentier becomes more prudent, the slope of the consumption profile becomes less negative until, at a critical value $\hat{\gamma}$, the slope changes sign; for $\gamma>\hat{\gamma}$, the consumption profile slopes upwards. ${ }^{8}$ In this latter case, the two precautionary effects identified in section 3 outweigh the hazard effect.

\section{A Review of Two Key Assumptions}

This section discusses briefly two of the assumptions that have been made in the analysis: (i) i.i.d. processes for asset returns and labour income, and (ii) $\rho=\delta$ i.e. no relative impatience.

The lack of persistence in the risky processes is unlikely to be empirically justifiable, particularly for labour income. There are three reasons for maintaining this assumption. First, a persistent process can, arguably, be approximated over a finite

\footnotetext{
${ }^{8}$ The familiar analytical result in e.g. Blanchard and Fischer (1989) means that $\hat{\gamma}=1$, i.e. $\log$ utility. While the approximation clearly demonstrates the existence of a critical value of $\gamma$, the bias in the approximation due to the omission of squared deviation terms means that it is not possible to show that $\hat{\gamma}=1$ exactly.
} 
horizon by a higher variance i.i.d. process; see e.g. Skinner (1988) and Zeldes (1989). Secondly, the focus of this paper is on the effect of uncertainty, separate from the issue of persistence. An important issue for further research is whether persistence in asset returns has a different effect on optimal consumption to persistence in labour income. For this initial analysis, however, we think it sufficient to consider whether uncertainty over the two processes is homogeneous. Finally, in an extended model (details of which are available from the authors on request), persistence has been incorporated in the analysis. The cost of doing so is a loss in tractability of the analytical solution for optimal consumption, and consequently of intuition.

The other restrictions in the i.i.d. assumption are that the processes are arithmetic and uncorrelated. It would be more normal to treat $r$ and $y$ as lognormally distributed, to avoid negative values. But since the approximation requires that the deviations from the riskless solution are small in percentage terms, the problem is not important. Correlation can be included with no significant change in the results.

The assumption of no relative impatience simplifies the analysis considerably: it means that the riskless consumption level $c^{*}$, and so the expansion point of the Taylor approximation, is constant over time. The analysis can be extended to the more general case of $\rho \neq \delta$; complications then arise in distinguishing shifts in the optimal consumption profile due to different source of uncertainty, and due to impatience. In the special case of CRRA utility, however, the various effects can be separated cleanly. Details of the extension are available from the authors on request.

\section{Numerical Solution for Optimal Consumption}

While the discussion of previous sections provides some insights, a more detailed examination of the impact of the two forms of uncertainty requires numerical solution of the model. This is provided in this section. There are two objectives for the discussion. The first is to examine and understand the qualitative aspects of the response of expected time profile of optimal consumption to uncertainty. The second is to assess the accuracy of the approximate solution which was derived in the previous section. 
The results in this section use the following parameters values: $T=10, \delta=\rho=1.4$ (so that $\theta=0.4),{ }^{9} a_{0}=0$ and $\hat{y}_{t}=0.5$ while labour income is received. Asset returns and labour income are assumed to be independent, normally distributed random variables:

$$
\begin{aligned}
& r_{t+1}=\delta+\epsilon_{r, t+1}, \quad \epsilon_{r, t+1} \sim N\left(0, \sigma_{r}^{2}\right), \\
& y_{t+1}=\hat{y}_{t}+\epsilon_{y, t+1}, \quad \epsilon_{y, t+1} \sim N\left(0, \sigma_{y}^{2}\right) .
\end{aligned}
$$

The consumer receives labour income for the first seven periods of life; at this stage, she retires, and receives no labour income for the remaining 3 periods. (This is a very simple case of a life cycle earnings profile.) The utility function is CRRA.

\subsection{Qualitative Features of Optimal Consumption}

Three sets of results are presented in this subsection:

1. Labour income uncertainty (with $\sigma_{y}=0.1$ ); no asset return uncertainty $\left(\sigma_{r}=\right.$ $0)$.

2. No labour income uncertainty $\left(\sigma_{y}=0\right)$; asset return uncertainty (with $\sigma_{r}=$ $0.1)$.

3. Labour income uncertainty (with $\sigma_{y}=0.1$ ); asset return uncertainty (with $\left.\sigma_{r}=0.1\right)$.

For each set of results, four values of the CRRA parameter are used: $\gamma=-1$ (quadratic), 1 (logarithmic), 2, and 5. This range is intended to accommodate levels of risk aversion which have been studied analytically (such as the quadratic case), and which have been estimated empirically.

The results are shown in figures 1-3. The horizontal axis measures time, the vertical the percentage deviation of consumption from the riskless level (i.e. $C_{t}$ ). The

\footnotetext{
${ }^{9}$ The high value of $\theta$ reflects the low number of periods. If each period corresponds to roughly 5 years, this value of $\theta$ gives an annual expected asset return of around $7 \%$, which is about right for equities.
} 
level of consumption is the unconditional expectation of consumption over the life cycle, with expectations taken in the first period. Each figure has two lines. The solid line is the outcome of a grid numerical method used to solve the general Euler equation (4). The method used is standard, and is described in e.g. Zeldes (1989) and Deaton (1992). ${ }^{10}$ This will be referred to as 'the exact solution'. The dashed line (which is not always visible, since often it lies very close to the solid line) is the linear solution (14) from the Taylor approximation to the Euler equation. This will be referred to as 'the Taylor solution'.

Figure 1 shows the time profiles of consumption for the exact and Taylor solutions when only labour income is uncertain. When $\gamma=-1$, certainty equivalence holds, and both solutions return zero i.e. the level of consumption equals the riskless level. As $\gamma$ increases, the consumption profiles in both solutions slope upwards while the consumer receives labour income (i.e. for the first seven periods). This is the familiar effect of the precautionary motive. Note that the slopes of the consumption profiles increase with $\gamma$. During the retirement phase, the consumption profiles are flat, since the consumer faces no uncertainty in this case.

Figure 2 shows the time profiles of consumption when only asset returns are uncertain. When $\gamma=-1$, all precautionary effects are zero. The income profile used in this section means that savings are positive; the hazard effect therefore induces a downward slope to the consumption profiles. While labour income is positive (in the first seven periods, before retirement), the consumption profiles are downward sloping for all values of $\gamma$. In other words, the hazard effect always dominates. But as $\gamma$ increases, the precautionary covariance effect increase in importance, and so the slopes of the consumption profiles decrease in magnitude. Note that, for $\gamma>1$, the profiles slope upward during the retirement phase. This can be understood by noting that the consumer is essentially a rentier during retirement - deriving income only from the risky returns of the asset. Since savings are negative during the retirement phase, the hazard effect imparts a downward slope to the consumption profiles. This is offset by precautionary covariance effect when $\gamma>-1$. When $\gamma<1$, the hazard effect dominates, and the profiles are downward sloping during retirement. Analyt-

\footnotetext{
${ }^{10}$ The programs for the numerical solution were written in GAUSS, and are available from the authors on request.
} 


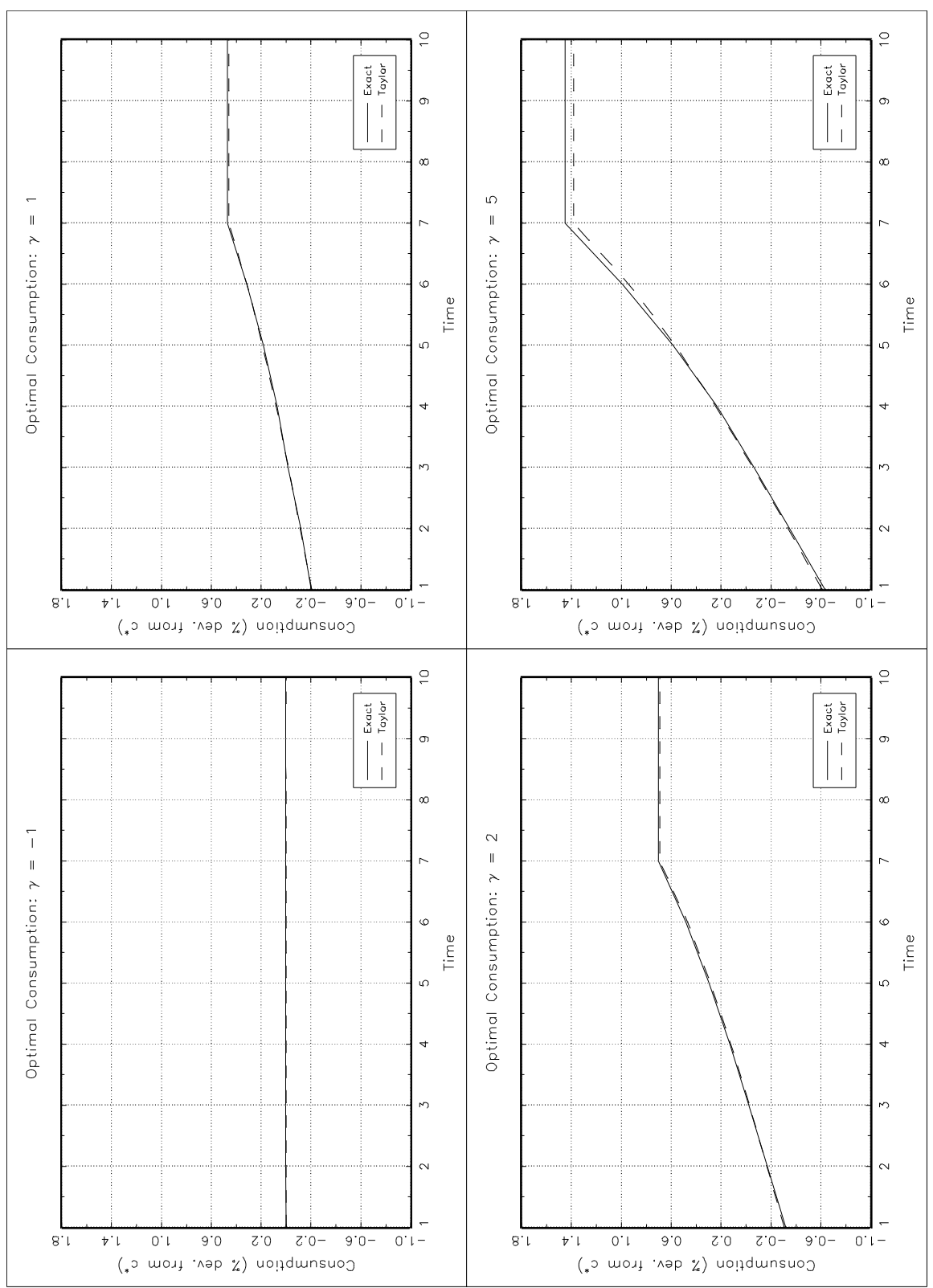

Figure 1: Optimal Consumption: $\sigma_{y}=0.1$ and $\sigma_{r}=0$ 
ically, the various effects balance exactly when $\gamma=1$. This result is reflected in the exact solution, which has a flat profile during retirement. The Taylor solution is less accurate, and has a (slightly) downward sloping profile during this phase. There is, however, a distinct change in the slope of the Taylor solution at $t=7$. For higher $\gamma$, both solutions have upward sloping consumption profiles during the retirement phase. $^{11}$

Finally, figure 3 shows the time profiles of consumption when there is uncertainty about both labour income and asset returns. Note that certainty equivalence does not hold when $\gamma=-1$, due to asset return risk. Since all precautionary effects are zero at this value of $\gamma$, only the hazard effect is important; hence the solution is identical to the case with pure asset return uncertainty. With positive saving (as there is in this case), the consumption profiles are downward sloping. As $\gamma$ increases, the precautionary effects grow in importance. Consequently, for $\gamma \geq 1$, the consumption profiles are upward sloping over their entire range. As in the previous cases, there is a distinct change in the slopes of the consumption profiles at retirement, as the consumer becomes a rentier.

\subsection{A Comparison of the Approximation and Exact Numerical Results}

Figures 1-3 indicate that the Taylor solution is a reasonable approximation to the exact solution, for the range of parameters examined. Recall that the Taylor approximation is based on the assumption that percentage deviations from the riskless solution are small (equation (12)). The figures indicate that this assumption is satisfied; for example, when $\sigma_{y}=0.1, \sigma_{r}=0$ and $\gamma=2$, the maximum percentage deviation of consumption is around $3 \%$, so that the squared percentage deviation is around $0.09 \%$. Even when the percentage deviation of optimal consumption is relatively large, the approximation performs reasonably well. For example, when $\sigma_{y}=\sigma_{r}=0.1$ and $\gamma=5$, the maximum percentage deviation is around $4.8 \%$; the difference between the exact and Taylor solutions at this point is less than $0.1 \%$. In

\footnotetext{
${ }^{11}$ The inaccuracy of the Taylor solution means that, during retirement, there is a range of $\gamma$ slightly larger than 1 over which the exact consumption profile has an upward slope, while the Taylor consumption profile has a downward slope.
} 


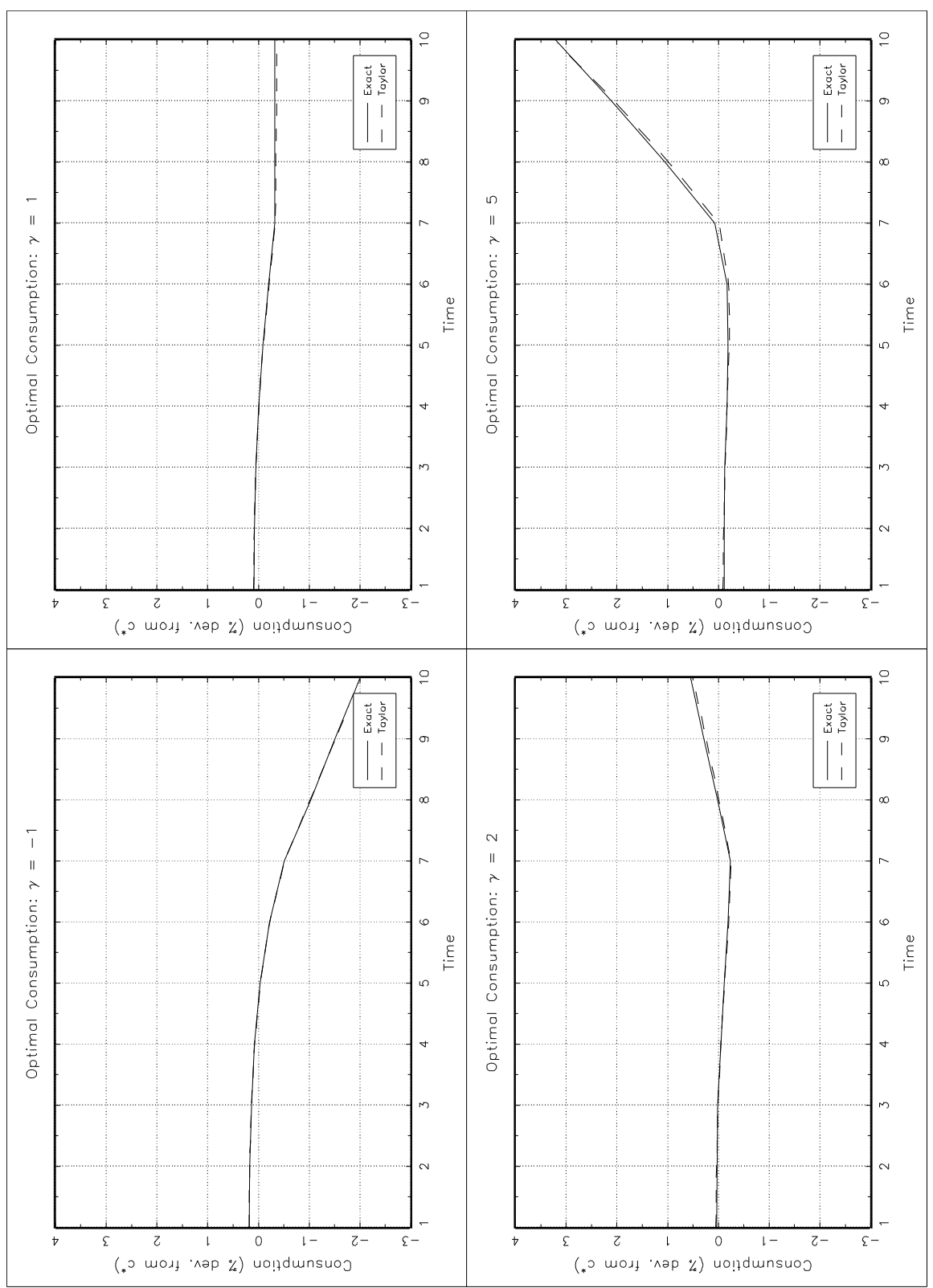

Figure 2: Optimal Consumption: $\sigma_{y}=0$ and $\sigma_{r}=0.1$ 


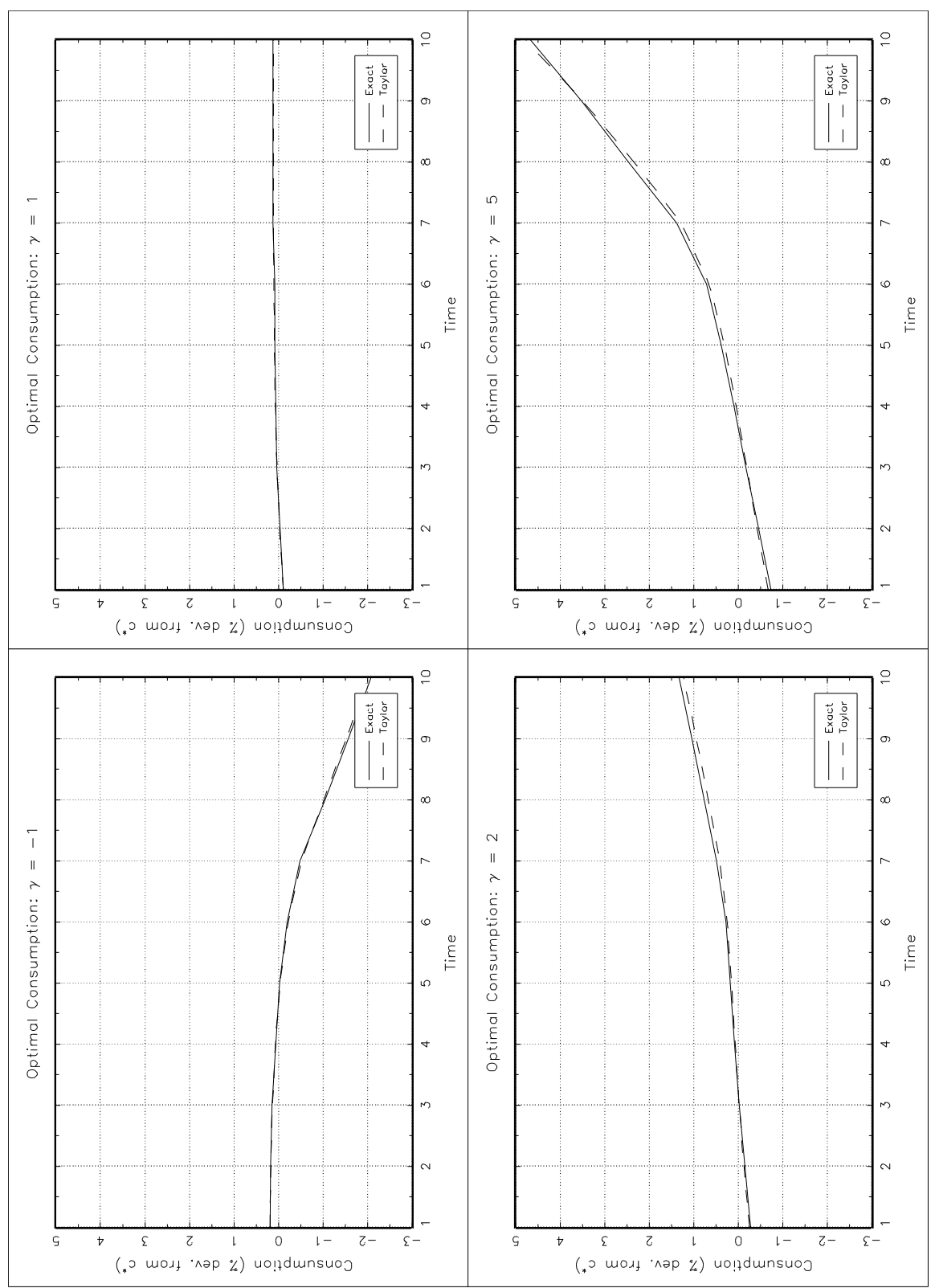

Figure 3: Optimal Consumption: $\sigma_{y}=\sigma_{r}=0.1$ 
all cases examined above, the Taylor solution has the same qualitative features as the exact. For example, when $\sigma_{y}=0, \sigma_{r}=0.1$ and $\gamma=5$, the difference between exact and Taylor solutions is around $8 \%$ (percentage deviations of $1.05 \%$ and $0.97 \%$ respectively); but the Taylor series captures well the overall shape of the exact consumption function.

Naturally, there are parameter values for which the closed form approximation is not accurate. Most crucially, the approximation fares badly at high variances and values of the CRRA parameter. ${ }^{12}$ Figure 4 shows the exact and Taylor solutions when $\sigma_{y}=\sigma_{r}=0.3$ i.e. when the annual variances of the labour income and asset return processes are around $11 \%$. For small $\gamma$, the quantitative difference between the solutions is small. As $\gamma$ increases, however, the gap between the exact and Taylor solutions grows; with $\gamma=5$, the exact solution is as much as four times larger than the Taylor solution. Notice that the small percentage deviations assumption of equation (12) is violated for higher values of $\gamma$ : optimal consumption in the exact solution departs from the riskless level by over $100 \%$ when $\gamma=5$. (For values of $\gamma$ above 5 and higher variances, there were convergence problems with the numerical routines.)

\subsection{Summary}

The purpose of this section has been to examine further the properties of the linear solution (14); and to assess the accuracy of the approximation. The numerical analysis has confirmed the three effects (standard precautionary, hazard, and precautionary covariance) identified in previous sections. It has also suggested that the Taylor approximation is quite reasonable. For moderate variances and CRRA parameters, there is no qualitative disagreement between the Taylor and exact solutions; and often small quantitative differences between the two. ${ }^{13}$

\footnotetext{
${ }^{12}$ The performance of the approximation has been assessed over different values of $T, \delta$ and the income profile $\left\{\hat{y}_{t}\right\}$. The approximation performs less well quantitatively as $T$ and $\delta$ increase; variations in the income profile appear to make little difference. The variances and the CRRA parameter are by far the most important variables for the accuracy of the closed form solution.

${ }^{13}$ There may be an additional benefit to our approach: for appropriate parameter values, it might allow optimal consumption to be calculated without resorting to computationally-intensive grid
} 


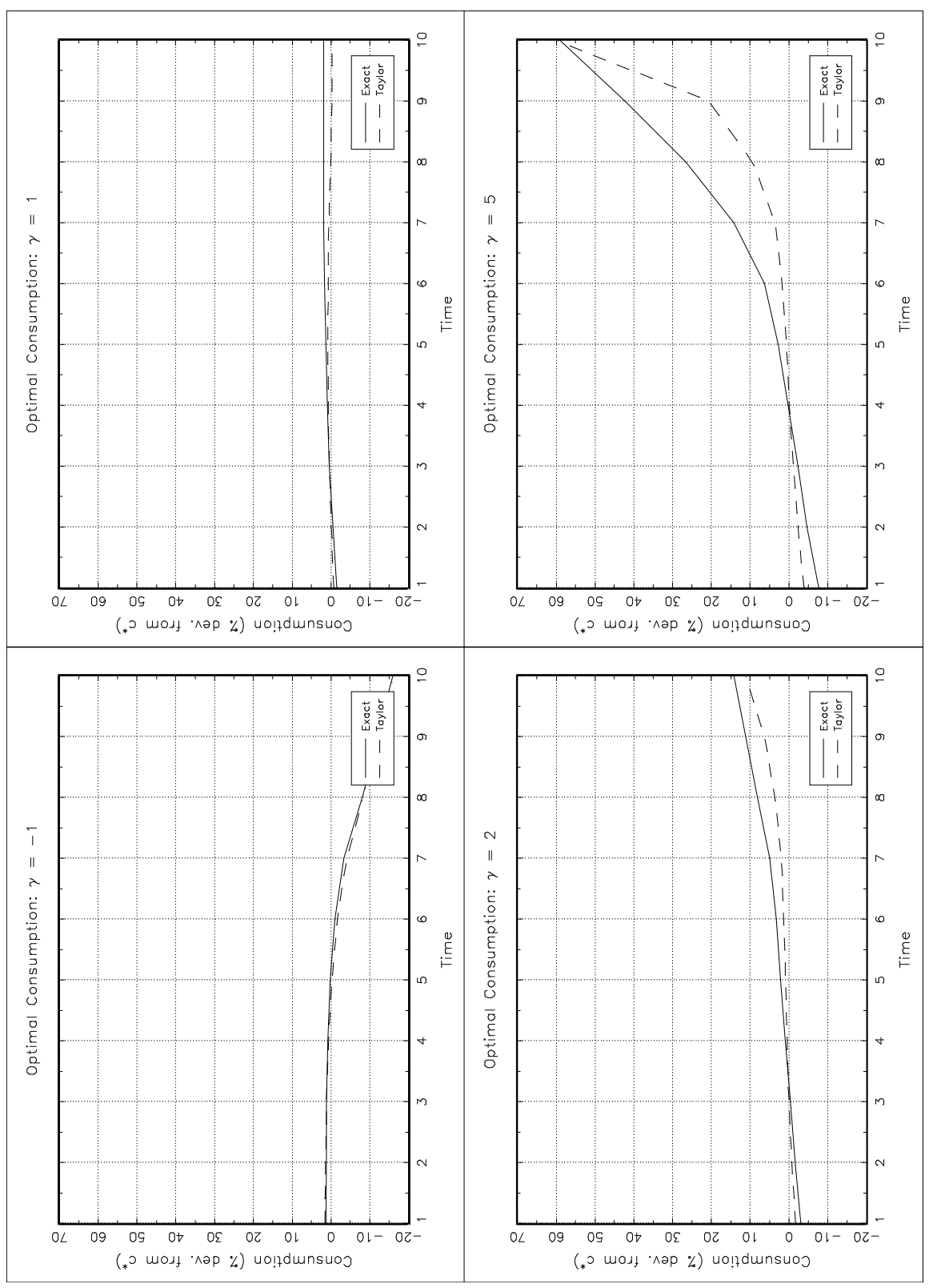

Figure 4: Optimal Consumption: $\sigma_{y}=\sigma_{r}=0.3$ 


\section{ConClusions}

The difficulties in solving the Euler equation limit the analysis of intertemporal consumption under uncertainty. Much previous work has concentrated on the case of stochastic labour income, but deterministic asset returns. The assumption has been that asset return risk will make no qualitative difference to the results. We argue in this paper that this assumption is wrong. Our objective has been to provide a solution to the Euler equation which allows economic intuition to be developed about the various effects that different types of uncertainty may have on optimal consumption. The quadratic and linear approximations, and the resulting closed form expression for consumption, achieve this. Three major results arise from the analysis:

1. Asset return and labour income uncertainty have qualitative different effects on optimal consumption.

2. The response of optimal consumption to uncertainty can be decomposed into three effects. The first is the familiar precautionary motive towards labour income uncertainty; see e.g. Deaton (1992). The second is a 'hazard effect', which induces a net saver to engage in less intertemporal consumption smoothing in response to asset return uncertainty. Finally, there is a 'precautionary covariance' effect, which arises only when there is asset return uncertainty. The precautionary covariance effect may offset or add to the other effects.

3. When there is only labour income uncertainty, the difference between current and next period's expected consumption does not depend on current assets. ${ }^{14}$ With asset return uncertainty, cash-in-hand enters the Euler equation; and so the expected change in consumption is a function of current assets.

It is common in work on macroeconomic policy-making to posit a policy loss

methods. For the analysis in section 6 , the grid solutions took up to four hours to calculate; in contrast, the closed form solution from the Taylor approximation took around 0.01 seconds to calculate. Of course, before the approximation can be used as any sort of substitute for more intensive numerical methods, it is necessary to conduct a more thorough assessment of its performance.

${ }^{14}$ Alternatively, the deviation of consumption under uncertainty from its riskless level is independent of assets. 
function with the variances of output and inflation as arguments. The framework of this paper suggests a natural basis from which to derive such a loss function, with inflation uncertainty proxied by non-diversifiable rate of return uncertainty, and output uncertainty proxied by non-diversifiable labour income uncertainty. ${ }^{15}$ It is fairly straightforward to use the analytical results of the paper to derive variances of the representative agent's consumption at all horizons. It is also easy to show that an approximation to the value function can be derived as a function of these variances, and the expected squared deviations from the risk-free case. By positing a specific functional form for utility (the most obvious being the CRRA form), therefore, a metric of the welfare costs of both forms of uncertainty can be derived, as functions of the underlying variances and the coefficient of relative risk aversion.

Finally, it would be interesting to examine more closely the possibility that asset return uncertainty might have effects beyond those of simply increasing variances. We have shown here that one clear impact of asset return uncertainty is to deter borrowing when cash-in-hand is below the risk-free consumption level. This might occur during e.g. a human capital formation phase of the life cycle. But, since future expected labour income is treated as exogenous in the model here, reduced borrowing reduces consumption, and not human capital formation. Relaxing the assumption of labour income exogeneity might therefore allow for asset return uncertainty to affect the equilibrium level, as well as variance, of consumption.

\footnotetext{
${ }^{15}$ Indeed, the supposition that both types of uncertainty in the analytical model are nondiversifiable seems to accord well with the notion that they arise from macroeconomic, and hence systemic, rather than individual-specific risk.
} 


\section{APPENDIX}

LemmA A.1: The covariance term $\operatorname{Cov}\left[U^{\prime}\left(c_{t+1}\right), r_{t+1}\right]$ has the opposite sign to net saving $\left(x_{t}-c_{t}\right)$, when $r_{t+1}$ and $y_{t+1}$ are independent random variables.

Proof: The covariance is

$$
\int_{\mathcal{Y}}\left[\int_{\mathcal{R}}\left(U^{\prime}(c)-\mathbb{E}\left[U^{\prime}(c)\right]\right)(r-\delta) f^{r}(r) d r\right] f^{y}(y) d y
$$

where $\mathcal{Y}$ is the set of permissible values of the random variable $y$ and $\mathcal{R}$ the set of permissible values of the random variable $r ; f^{r}(r)$ and $f^{y}(y)$ are the marginal probability density functions of the random variables $r$ and $y$; and time subscripts have been omitted for clarity. Note that $c$ is a random variable, since optimal consumption in period $t+1$ will be a function of realised cash-in-hand in period $t+1$, which is determined by the random variables $r$ and $y$ :

$$
c_{t+1}=g_{t+1}\left(\left(x_{t}-g_{t}\left(x_{t}\right)\right) r_{t+1}+y_{t+1}\right)
$$

where $g_{t+1}($.$) is the optimal consumption function in period t+1$. Note also that

$$
\mathbb{E}\left[U^{\prime}(c)\right]=\int_{\mathcal{Y}} \int_{\mathcal{R}} U^{\prime}(c) f^{r}(r) f^{y}(y) d r d y
$$

Now, $\mathbb{E}\left[U^{\prime}(c)\right]$ can be written as $U^{\prime}(\mathbb{E}[c])+J$, where $J$ represents a 'Jensen's inequality' term which is strictly positive when marginal utility is strictly convex. Define the inner integral in equation (A1) as

$$
\begin{aligned}
I & \equiv \int_{\mathcal{R}}\left(U^{\prime}(c)-\mathbb{E}\left[U^{\prime}(c)\right]\right)(r-\delta) f^{r}(r) d r \\
& =\int_{\mathcal{R}}\left(U^{\prime}(c)-U^{\prime}(\mathbb{E}[c])\right)(r-\delta) f^{r}(r) d r+\int_{\mathcal{R}} J(r-\delta) f^{r}(r) d r \\
& =\int_{\mathcal{R}}\left(U^{\prime}(c)-U^{\prime}(\mathbb{E}[c])\right)(r-\delta) f^{r}(r) d r .
\end{aligned}
$$

Define

$$
\begin{aligned}
& \alpha_{1}(r) \equiv U^{\prime}(c)-U^{\prime}(\mathbb{E}[c]), \\
& \alpha_{2}(r) \equiv r-\delta .
\end{aligned}
$$


Clearly, $\alpha_{2}(\delta)=0$, and $\alpha_{2}^{\prime}(r)>0 . \alpha_{1}(\delta)=0$, since $c=\mathbb{E}[c]$ when $r=\delta$ (for any given $y$ ). To determine the derivative of $\alpha_{1}$ with respect to $r$, it is helpful to show time subscripts explicitly:

$$
\begin{aligned}
\alpha_{1}\left(r_{t+1}\right) & \equiv U^{\prime}\left(c_{t+1}\right)-U^{\prime}\left(\mathbb{E}\left[c_{t+1}\right]\right) \\
& =U^{\prime}\left(g_{t+1}\left(\left(x_{t}-c_{t}\right) r_{t+1}+y_{t+1}\right)\right)-U^{\prime}\left(\mathbb{E}\left[g_{t+1}\left(\left(x_{t}-c_{t}\right) r_{t+1}+y_{t+1}\right)\right]\right)
\end{aligned}
$$

Hence $^{16}$

$$
\frac{\partial \alpha_{1}}{d r_{t+1}}=U^{\prime \prime}\left(x_{t}-c_{t}\right) \frac{\partial g_{t+1}}{\partial x_{t+1}}
$$

$U^{\prime \prime}<0$, since the consumer is assumed to be risk averse. To determine the sign of $\frac{\partial g_{t+1}}{\partial x_{t+1}}$, consider the Bellman equation of optimality for period $t+1$ :

$$
V_{t+1}\left(x_{t+1}\right)=\max _{c_{t+1}}\left(U\left(c_{t+1}\right)+\frac{1}{\delta} \mathbb{E}\left[V_{t+2}\left(x_{t+2}\right)\right]\right)
$$

where $V_{t+1}$ is the period $t+1$ value function. The first order condition is

$$
U^{\prime}\left(c_{t+1}\right)-\frac{1}{\delta} \mathbb{E}\left[\frac{\partial V_{t+2}\left(x_{t+2}\right)}{\partial x_{t+2}} r_{t+2}\right] .
$$

Theorems 4.10 and 4.11 of Stokey and Lucas (1989, pp. 845) ensure continuous differentiability of the value function to this problem. Therefore differentiate the Bellman equation (A2) with respect to $x_{t+1}$ :

$$
\frac{\partial V_{t+1}}{\partial x_{t+1}}=\frac{1}{\delta} \mathbb{E}\left[\frac{\partial V_{t+2}}{\partial x_{t+2}} r_{t+2}\right]
$$

With equation (A3), this gives

$$
\frac{\partial V_{t+1}}{\partial x_{t+1}}=U^{\prime}\left(c_{t+1}\right)
$$

Therefore

$$
\frac{\partial^{2} V_{t+1}}{\partial x_{t+1}^{2}}=U^{\prime \prime} \frac{\partial c_{t+1}}{\partial x_{t+1}}
$$

\footnotetext{
${ }^{16}$ Note that this derivative uses the assumption that $r$ and $y$ are independently distributed. This assumption is stronger than is required to show that the covariance term has the opposite sign to $\left(x_{t}-c_{t}\right)$.
} 
Hence Sign $\left(\frac{\partial c_{t+1}}{\partial x_{t+1}}\right)=\operatorname{Sign}\left(\frac{V^{\prime \prime}}{U^{\prime \prime}}\right)$. The arguments of Grossman et al. (1979) and Neave (1971) can be adapted to show that $\operatorname{Sign} V^{\prime \prime}=\operatorname{Sign} U^{\prime \prime}$. Therefore $\frac{\partial c_{t+1}}{\partial x_{t+1}}>0$.

Consequently,

$$
\operatorname{Sign}\left(\frac{\partial \alpha_{1}}{d r_{t+1}}\right)=-\operatorname{Sign}\left(x_{t}-c_{t}\right)
$$

Therefore, if $\left(x_{t}-c_{t}\right)>0$ (i.e. the consumer is a net saver), $\frac{\partial \alpha_{1}}{d r_{t+1}}<0$. This means that $\alpha_{1}(r) \alpha_{2}(r) \leq 0 \forall r$; and so $I \leq 0$, and $\operatorname{Cov}\left[U^{\prime}\left(c_{t+1}\right), r_{t+1}\right] \leq 0$. (The converse holds.) Therefore the covariance term has the opposite sign to $\left(x_{t}-c_{t}\right)$.

Lemma A.2: The magnitude of the covariance term is locally decreasing in the degree of convexity of marginal utility.

Proof: Consider two different utility functions, $U$ and $V$, where both utility functions are strictly increasing, concave, and have positive third derivatives. Let $V^{\prime}=G\left(U^{\prime}\right)$, where $G^{\prime}<0, G^{\prime \prime}>0$, and $G\left(U^{\prime}(\mathbb{E}[c])\right)=U^{\prime}(\mathbb{E}[c])$. The shape of the function $G$ means that $V^{\prime}$ is more convex than $U^{\prime}$. (The third condition on $G$ is simply a normalisation.) Define

$$
\begin{aligned}
& I_{U}=\int_{\mathcal{R}}\left(U^{\prime}(c)-\mathbb{E}\left[U^{\prime}(c)\right]\right)(r-\delta) f^{r}(r) d r, \\
& I_{V}=\int_{\mathcal{R}}\left(V^{\prime}(c)-\mathbb{E}\left[V^{\prime}(c)\right]\right)(r-\delta) f^{r}(r) d r .
\end{aligned}
$$

The objective of the proof is to show that $\left|I_{V}\right| \leq\left|I_{U}\right|$.

Let

$$
\Delta I \equiv I_{U}-I_{V}=\int_{\mathcal{R}}\left(U^{\prime}(c)-V^{\prime}(c)\right)(r-\delta) f^{r}(r) d r
$$

(Note that the terms in $\mathbb{E}\left[U^{\prime}(c)\right]$ and $\mathbb{E}\left[V^{\prime}(c)\right]$ drop out, since e.g. $\int_{\mathcal{R}} \mathbb{E}\left[U^{\prime}(c)\right](r-\delta) f^{r}(r) d r=$ $\mathbb{E}\left[U^{\prime}(c)\right] \int_{\mathcal{R}}(r-\delta) f^{r}(r) d r=0$.) Define

$$
\begin{aligned}
& \alpha_{1}(r)=U^{\prime}(c)-V^{\prime}(c), \\
& \alpha_{2}(r)=r-\delta .
\end{aligned}
$$


$\alpha_{2}(\delta)=0$, and $\alpha_{2}^{\prime}(r)>0 . \alpha_{1}(\delta)=U^{\prime}(\mathbb{E}[c])-V^{\prime}(\mathbb{E}[c])=0$. The derivative of $\alpha_{1}$ is

$$
\begin{aligned}
\alpha_{1}^{\prime}(r) & =U^{\prime \prime}\left(x_{t}^{U}-c_{t}^{U}\right) \frac{\partial c_{t+1}^{U}}{\partial x_{t+1}}-V^{\prime \prime}\left(x_{t}^{V}-c_{t}^{V}\right) \frac{\partial c_{t+1}^{V}}{\partial x_{t+1}} \\
& =U^{\prime \prime}\left(x_{t}^{U}-c_{t}^{U}\right) \frac{\partial c_{t+1}^{U}}{\partial x_{t+1}}\left(1-G^{\prime} \frac{\left(x_{t}^{V}-c_{t}^{V}\right) \frac{\partial c_{t+1}^{V}}{\partial x_{t+1}}}{\left(x_{t}^{U}-c_{t}^{U}\right) \frac{\partial c_{t+1}^{U}}{\partial x_{t+1}}}\right) .
\end{aligned}
$$

Note that the consumption function, and consequently wealth, will depend on the utility function; the variables are therefore superscripted with the relevant utility function. When $G$ is a small transformation (in the sense that $\left|\frac{G^{\prime \prime}}{G^{\prime}}\right|$ is $\operatorname{small}$ ), then $\operatorname{Sign}\left(x_{t}^{U}-c_{t}^{U}\right)=\operatorname{Sign}\left(x_{t}^{V}-\right.$ $\left.c_{t}^{V}\right)$. Since $G^{\prime}<0$, Sign $\left(\alpha_{1}^{\prime}(r)\right)=-\operatorname{Sign}\left(x_{t}^{U}-c_{t}^{U}\right)$. When $\left(x_{t}^{U}-c_{t}^{U}\right)>0, \alpha_{1}^{\prime}(r)<0$, and $\alpha_{1}(r) \alpha_{2}(r) \leq 0 \forall r$. In this case, $\Delta I \leq 0$; and so $0 \geq I_{V} \geq I_{U}$. When $\left(x_{t}^{U}-c_{t}^{U}\right)<0$, $\alpha_{1}^{\prime}(r)>0$, and $\alpha_{1}(r) \alpha_{2}(r) \geq 0 \forall r$. In this case, $\Delta I \geq 0$; and so $0 \leq I_{V} \leq I_{U}$. Therefore $\left|I_{V}\right| \leq\left|I_{U}\right|$. Hence the magnitude of the covariance term is decreasing in the degree of convexity of marginal utility. 


\section{REFERENCES}

Binder, M., M. H. Pesaran and S. H. Samiei, (1998): "Analytical and Numerical Solution of Finite-Horizon Nonlinear Rational Expectations Models", Department of Applied Economics Working Paper, No. 9808.

Blanchard, O. J., (1985): "Debt, Deficits, and Finite Horizons", Journal of Political Economy, 93(2), 223-47.

Blanchard, O. AND S. Fischer, (1989): Lectures on Macroeconomics, Cambridge, Mass., MIT Press.

Caballero, R. J., (1991): "Earnings Uncertainty and Aggregate Wealth Accumulation", American Economic Review, 81, 859-871.

Carroll, C. D., (1997): "Death to the Log-Linearized Consumption Euler Equation! (And Very Poor Health to the Second-Order Approximation)", NBER Working Paper No. W6298.

Carroll, C. D. and M. S. Kimbal, (1996): "On the Concavity of the Consumption Function", Econometrica, 64(4), 981-92.

Deaton, A. S., (1991): "Saving and Liquidity Constraints", Econometrica, 59, 12211248.

Deaton, A. S., (1992): Understanding Consumption, Oxford, Clarendon Press.

Greene, W. H., (1993): Econometric Analysis, Prentice Hall, Second edition.

Grossman, D., D. Levhari and L. J. Mirman, (1979): "Consumption under Uncertainty", in Green, J. R. AND J. ScheinkMAN, (eds.), General Equilibrium, Growth and Trade: Essays in Honor of Lionel McKenzie, New York: Academic Press.

HaLl, R. E., (1978): "Stochastic Implications of the Life-Cycle/Permanent Income Hypothesis: Theory and Evidence", Journal of Political Economy, 96, 971-987.

Leland, H. E., (1968): "Savings and Uncertainty: The Precautionary Demand for Savings", Quarterly Journal of Economics, 82, 465-73.

Miles, D., (1997): "A Household Level Study of the Determinants of Incomes and Consumption" Economic Journal, 107(440), 1-25.

Neave, E. H., (1971): "Multiperiod Consumption-Investment Decisions and Risk Preferences", Journal of Economic Theory, 3(1), 40-53.

Siegel, J. J., (1992): "The Real Rate of Interest from 1800-1990: A Study of the U.S. and the U.K.", Journal of Monetary Economics, 29(2), 227-52. 
SkInNER, J., (1988): "Risky Income, Life-cycle Consumption, and Precuationary Saving", Journal of Monetary Economics, 22, 237-255.

Stokey, N. L. And R. E. Lucas, JnR., (1989): Recursive Methods in Economic Dynamics, Harvard University Press.

Talmain, G., (1998): "Exact and Approximate Solutions to the Problem of Precautionary Saving", Journal of Economic Dynamics and Control, 1998, 23(1), 113-124.

VAN DER Ploeg, F., (1993): "A Closed-Form Solution for a Model of Precautionary Saving", Review of Economic Studies, 60(2), 385-95.

WeIL, P., (1993): "Precautionary Savings and the Permanent Income Hypothesis", Review of Economic Studies, 60(2), 367-83.

ZELdes, S., (1989): "Optimal Consumption with Stochastic Income: Deviations from Certainty Equivalence", Quarterly Journal of Economics, 104, 275-298. 